\title{
Supercontinuum, solitons, and instabilities
}

\author{
Goëry Genty ${ }^{1}$ and John M. Dudley ${ }^{2}$ \\ ${ }^{1}$ Department of Physics, Tampere University of Technology, P. O. Box 692, FI-33101, Tampere, Finland \\ ${ }^{2}$ Institut FEMTO-ST, UMR 6174 CNRS-Université de Franche-Comté, Besançon, France \\ goery.genty@tut.fi
}

\begin{abstract}
Summary: Understanding the complex physical phenomena behind the generation of broadband supercontinuum light sources has enabled new technological developments and major fundamental contributions in many different fields of science.
\end{abstract}

The development of high brightness spatially-coherent light sources has enabled diverse applications in various areas ranging from spectroscopy and communications to high resolution imaging and material processing. A continual challenge, however, is to develop laser sources at new wavelengths tailored to specific applications. The primary approach to generate new wavelengths has been to develop new laser materials, but the wavelengths that can be emitted are generally limited by the intrinsic material properties.

Another approach is to use the processes of nonlinear optics to convert laser radiation from one wavelength to another. The last decade has seen a veritable revolution in this field with the development of the fiber supercontinuum, a truly remarkable source of optical radiation that can emit coherent light with high brightness spanning the visible and near infrared spectral regions [1,2]. Areas where supercontinuum sources are finding significant commercial success include optical frequency metrology, spectroscopy, and in particular microscopy where the high brightness and large bandwidth have greatly increased the achievable resolution compared to that obtained with traditional lamps or LED sources.

Generating a supercontinuum is relatively easy and only requires injection of intense pulses of light from a pump laser into an optical fiber with appropriate dispersion characteristics [3]. A supercontinuum generated by injecting femtosecond pulses into a highly nonlinear photonic crystal fiber is shown in Figure 1. Although generating a supercontinuum is rather straightforward, the physics of supercontinuum generation is much more involved. In short, it is based on inducing a nonlinear electronic polarization in a dielectric medium using intense pulses of light from a high power laser. If the intensity of the injected pulses into the medium is high enough, the refractive index of the medium can be modified in proportion to the incident intensity, a phenomenon commonly referred to as the optical Kerr effect. This change in the refractive index in turn induces a time-dependent phase shift or self-phase modulation across the temporal pulse that generates new wavelength components [4]. Explaining fiber supercontinuum generation in terms of only self-phase modulation, however, is an oversimplification and the rich physics that underpins the generation of a broadband supercontinuum truly arises from the complex interaction between the nonlinear self-phase modulation and the linear dispersion.

A significant effort has been put into modelling of the supercontinuum generation process. It is the advance in the development of accurate pulse propagation models that has led to a full understanding of supercontinuum dynamics [5], unraveling e.g. the central role played by soliton structures that manifest as a result of interplay between linear dispersion and selfphase modulation. In fact, the concepts of soliton physics are essential to obtain a correct 
physical picture of supercontinuum generation $[5,6]$. The wide range of accessible regimes where subtle soliton dynamics can occur continues to throw up surprises, revealing unexpected analogues of phenomena usually found in seemingly completely different systems such as the emergence of instabilities and rogue waves [7] and opening up new perspectives to study analogies with other areas of physics.

The ability to understand the complex physical phenomena behind supercontinuum generation has enabled compact tabletop systems with reduced power demands, leading to new technological developments and major contributions in many different fields of science. As new materials and laser pump sources continue to become available, the supercontinuum can be expected to continue finding new areas of applications, especially in the UV or in the mid-IR, and we can anticipate that some of them will be as unexpected and as exciting as the many that we have seen to date.

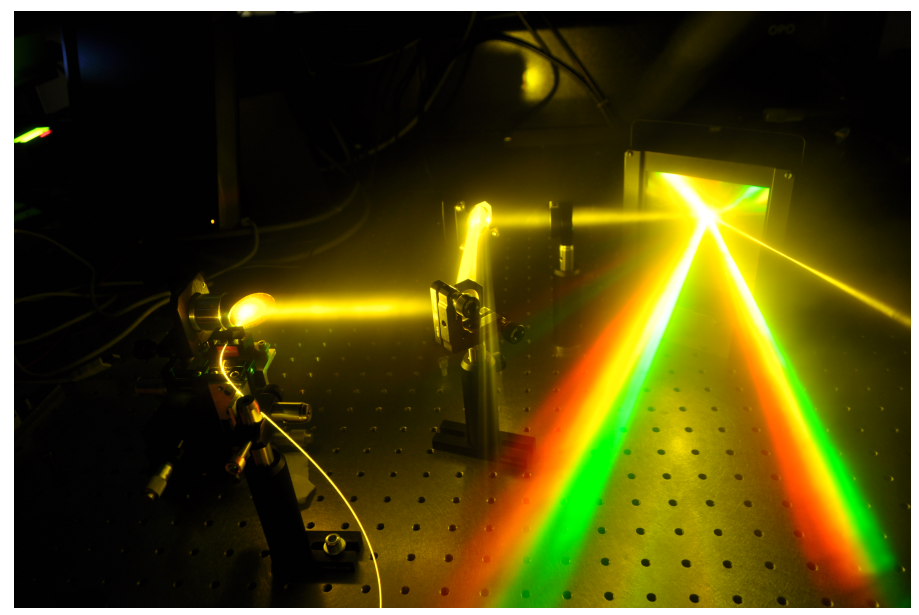

Figure 1. Supercontinuum generated by injecting femtosecond pulses from a Ti:Sapphire laser at 800 $\mathrm{nm}$ into a photonic crystal fiber. The supercontinuum is dispersed using a diffraction grating onto a screen. Although we see only the visible portion of the spectrum, the supercontinuum actually extends from below $500 \mathrm{~nm}$ into the infrared beyond $1500 \mathrm{~nm}$.

\section{References}

[1] The Supercontinuum Laser Source: Fundamentals with Updated References. Ed. R. R. Alfano, Springer (2006)

[2] Supercontinuum Generation in Optical Fibre. Eds J. M. Dudley and J. R. Taylor. Cambridge University Press (2010)

[3] J. K. Ranka, R. S. Windeler, and A. J. Stentz, Visible continuum generation in air-silica microstructure optical fibers with anomalous dispersion at $800 \mathrm{~nm}$, Opt. Lett. 25, 25-27 (2000).

[4] Nonlinear Fiber Optics. G. P. Agrawal, Fifth Edition, Academic Press (2012)

[5] J. M. Dudley, G. Genty, and S. Coen, Supercontinnum generation in photonic crystal fiber, Rev. Mod. Phys. 78, 1135-1184 (2006).

[6] Dmitry V. Skryabin and Andrey V. Gorbach, Colloquium: Looking at a soliton through the prism of optical supercontinuum, Rev. Mod. Phys. 82, 1287-1299 (2010)

[7] J. M. Dudley, F. Dias, M. Erkintalo, and G. Genty, Instabilities, breathers and rogue waves in optics, Nature Photonics 8, 755-764 (2014). 